\title{
Isometric Muscle Strength, Flexion
}

National Cancer Institute

\section{Source}

National Cancer Institute. Isometric Muscle Strength, Flexion. NCI Thesaurus. Code C139216.

An assessment of muscle strength that measures the force produced by static muscle contraction during the bending movement between body parts. 